1st October 2019. Patients were subsequently asked about driving, where appropriate, and the junior medical team provided education on the national drug driving guidance.

Results All 18 patients:

- Had prescriptions of medications listed in the drug driving guidance

- There was no knowledge or documentation of whether a patient was a vehicle driver

5 patients admitted for end of life care were therefore excluded from further analysis. When the 13 patients were asked:

- 10 patients were not vehicle drivers

- 3 patients were vehicle drivers

The drug driving guidance was discussed within the department's service improvement meeting. An addendum on the clerking proforma was made to include whether the patient was a vehicle driver or not. If a patient was a vehicle driver it was highlighted on the joint MDT handover list, and if prescribed medication listed in the drug driving guidance, they were counselled on this. This was also communicated on the discharge summary for ongoing continuity of care.

Conclusion This project enabled joint collaborative work between doctors and the pharmacy team. It also highlighted the importance of not making assumptions about palliative care patients not driving a vehicle. Discussing the drug driving guidance with the medical team ensured that a patient was aware of the government guidance and ensuring public safety. A re-audit is planned in 2020 to ensure that this change in practice remains embedded.

\section{RECRUTING MND PATIENT RESEARCH PARTICIPANTS: I THOUGHT WE WOULD HAVE MORE}

Natalie Ayton, Naomi Seaton, Christina Faull. LOROS Hospice, Leicester

10.1136/spcare-2020-PCC.130

Introduction LOROS Hospice provides a service to all people with MND in Leicester, Leicestershire and Rutland from the point of their diagnosis and research is an integrated aspect of the services. We wished to offer patients the opportunity to participate in the COMMEND study, a randomised controlled study of commitment and acceptance therapy. Despite a first stage feasibility study no guidance was available to inform the likely number of eligible participants.

The aim of this work was to identify the potential number of patients that could be approached about the study and understand the differences between the total population and this sample and its implication for study feasibility assessments.

Methods The notes of all patients with MND who were registered at the Hospice were identified and mapped to the inclusion and exclusion criteria of the COMMEND study.

Results 70 patients were identified of whom only 19 (27\%) were eligible for the COMMEND study. The largest number (33\%) were excluded because of receiving nutritional and/or ventilator support. A further $20 \%$ were excluded because of cognitive issues and $6 \%$ did not speak English as a first language.

Conclusions That less than a third of patients were eligible for this relatively straightforward interventional study was a surprise to us. It is a well known aphorism that potential participants disappear as soon as a study opens. This work provides a screening analysis which informs realistic feasibility assessment and more nuanced understanding of the MND patient population. As LOROS provides care to all patients in the geographical area these findings should be generalizable.

\section{ONE HOSPITAL CLINIC - ACHIEVING DEATH AT HOME AND HOSPICE FOR PATIENTS WITH PULMONARY FIBROSIS}

Kirsten Baron, Sarah Simm, Matthew Craggs, Abdul Ashish. Wrightington Wigan and Leigh NHS Foundation Trust

\subsection{6/spcare-2020-PCC.131}

Background Pulmonary fibrosis is a chronic respiratory condition with a poor prognosis and significant symptom burden, yet has traditionally been managed purely by respiratory physicians. It was the experience in the Greater Manchester town of Wigan that many patients with pulmonary fibrosis were not recognised to be in the last year of life and often presented to and ultimately died in hospital during their final illness. There were missed opportunities for improving symptom control, advance care planning (ACP) and achievement of preferred place of death (PPD).

Methods A new service was established where a Palliative Medicine Consultant and clinical nurse specialist reviewed patients in a dedicated clinic running alongside the respiratory clinic. The monthly face to face clinic was complemented with a fortnightly telephone follow up clinic in-between. Symptom issues were addressed guided by Integrated Palliative care Outcome Scale (IPOS) completion, hand-held fans given out to help manage breathlessness, referrals considered to the local AHP-run palliative care out-patient centre and ACP and PPD discussed and recorded.

Results Since December 2017, to date 36 patients with Pulmonary Fibrosis have been referred to the clinic. Three patients died prior to review. Of the 33 who have been assessed, 9 remain under active follow-up, 6 have been discharged to community palliative care services, 1 back to GP and 17 have died. Advance care planning was initiated in 26 patients $(79 \%)$ and completed in $18(55 \%)$. Of the 17 patients that died, all but two died out of hospital (88\%), 11 in their own home/nursing home and 4 in hospice, significantly greater than national averages for non-malignant conditions.

Conclusion The development of a hospital-based dedicated palliative medicine clinic for patients with Pulmonary Fibrosis has resulted in a majority of out-of-hospital deaths for this group of patients.

\section{SURVEYING THE USERS OF AN OPEN-ACCESS SUPPORT SERVICE FOR PEOPLE AFFECTED BY AN ILLNESS THAT HAS NO CURE}

Ellen R Battye, Paul M Taylor. University of Sheffield, St Luke's Hospice

\subsection{6/spcare-2020-PCC.132}

Background In the UK, palliative care is a growing specialty; however, national inequalities in access to services persist. Those who are male, older, more deprived, belong to ethnic minority groups and have non-malignant disease are relatively 